\title{
A QOS-BASED RESOURCE RESERVATION PROTOCOL FOR PRIORITY-DIFFERENTIATED DELAY-BOUNDED MULTICAST
}

\author{
Longsong Lin $^{1}$, Ming-Shou Liu ${ }^{2}$, Lih-Chyau Wuu ${ }^{1}$ and Chun-Yeh Tsai ${ }^{1}$ \\ ${ }^{1}$ Dept. of Elec. and Info. Engr. \\ 2 Dept. of Elec. Engr. \\ Natl. Yunlin Univ. Sci. \& Tech. \\ Natl. Chinyi Inst. of Tech. \\ Touliu, Yunlin, Taiwan \\ Taiping, Taichung, Taiwan. \\ $\{$ lin, wuulc $\} @$ el.yuntech.edu.tw \\ \{liu\} @chinyi.ncit.edu.tw
}

\begin{abstract}
Discerning the diverging developments of Internet routing and resource reservation signaling protocols, we argue that when it comes to the overall efficiency and utilization of the network resources, the reservation signaling must collaborate with underly ing routing protocol. This paper presents a multicast QoS-based routing and reservation protocol (MQRP) that integrates resource reservation algorithm with multicast routing to guarantee end-to-end delay bounds for prioritydifferentiated multicast. MQRP allows each receiver to prioritize its selection on multiple hierarchical layer-encoded media streams and bounds the end-toend delays for the selected flows. Given the priority and delay constraints, this receiver-initiated protocol employs RSVP signaling messages and OSPF-based routing mechanism to establish a multicast tree and reserve link bandwidth, aiming at maximizing the gain and utilization of the netw ork. To compare protocols for solving the priority-differentiated, delay-bounded multicast problems, simulations are conducted on a class of random networks. It is shown that MQRP has best scalability to the number of network nodes as well as offered source streams. Furthermore, in case that the network resource is insufficient to convey all the requests, MQRP can admit the request with higher priorities so as to leverage the overall network utilization.
\end{abstract}

Keywords: Multicast algorithms, Resource reservation protocols, Multimedia multicast, QoS-based routing

\section{INTRODUCTION}

Motivated by the increasing demand on broadband multimedia applications as well as by the enriched functionality at users desktops nowadays, there is a need to provide multiple-point [1], guaranteed quality of services (QoS) [2][3] 
in a heterogeneous networking environment. Multicast promises the efficient use of the network bandwidth for multiple-point communication; nevertheless, delivering guaranteed multicast service is more than a simple capacity issue. It involves not only a routing protocol to establish QoS-assured paths but also a signaling procedure to secure the bandwidth for each individual, thus engineering towards optimal usage of network resources. To provide efficient resource usage while guar anteeing QoS routes, multicast routing and resource reservation protocols thus come to orchestrate naturally [1][4].

Resource reservation protocols and QoS-based routing, however, have evolved into separated efforts and neither an integrated discussion nor multicast context has been much discussed. ST-II and RSVP are among two resource reservation protocols proposed for supporting guaranteed services [5][6]. For both, the guaranteed quality of service is characterized by approximating a "fluid model" of service [5][7][8] in which a stream effectively sees a dedicated wire of the assigned bandwidth between the source and the de stination. The major difference between ST-II and RSVP lies in how multicast is supported within reservation signaling. In addition, RSVP is not equipped with any mechanism to instruct routing on QoS attributes; it is still an open issue how QoS is attained, and yet none of the current researches have yet sufficiently dealt with the interaction of routing and QoS guarantees [4]. Apart from ST-II and RSVP, QoS-based routing [9] approach, which does not include a resource reservation mechanism, allows the determination of a path that will possibly accommodating the requested QoS. Thereupon, paths for flows would be determined based on some knowledge of resource availability in the network, as well as the QoS requirement of flows.

The issues surrounding resource reservation and QoS routing for multicast have been centered at how to discover the status of resource usage in the current multicast. This status information could improve the feasibility of finding a path for a new receiver joining to a multicast flow during path computation. QOSPF [10] handles this problem by having routers broadcast reserved resource information to other routers. With the global information on residual bandwidth, each node in QOSPF can compute the path that satisfies a QoS request. However, in $\mathrm{m}$ ulticast case, this approach may not generate an optimal routing tree as it is not constructed by considering the receivers' requirements. To avoid broadcasting the information of resource availability as with QOSPF, alternate path routing [11] deals with this issue by using probe messages to find a path that has sufficient resources to fulfill the QoS requirements. Path QoS Computation (PQC) method, proposed in [12], uses RSVP PATH messages to propagate bandwidth allocation information. However, a router receiving the PATH message gets the knowledge of the resource allocation only on those links along the path from the source to itself. Allocation for the same flow on 
other remote branches of the multicast tree is not available. Thus, the PQC method may not be sufficient to find feasible paths to all receivers.

In this paper we present a multicast QoS-based routing and resource reservation protocol (MQRP) to support the priority-differentiated, delay-guaranteed multicast. The protocol allows a customer to specify different priorities on a set of selected streams from a source and set an end-to-end delay bounds for the requested streams. It integrates a link-state routing protocol with the RSVP bandwidth reservation protocol to establish the multicast tree and reserve tree bandwidth simultaneously. The goal in designing MQRP is to leverage the overall efficiency and utilization of the network.

Our approach distinguishes from previous works in the following respects. 1.) Dynamic determination of feasible paths initiated from receivers: The receiver-initiated MQRP avoids the broadcast storm as with QOSPF and adopts alternative path approach by dynamically establish the multicast tree from all receivers to the source. requirements is a more difficult 2.) Optimization of resource usage: $\mathrm{MQRP}$ scheme can improve the utilization and efficiency of network resources. It attempts to optimize the network resource utilization by minimizing the reserved bandwidth of the tree as well as by maximizing the total number of streams admitted to the ne twork, and to optimize the efficiency by admitting streams with higher gains. 3.) Path selection based on per-flow priority: MQRP allows a user to prioritize the selected streams and to specify a delay bound for these streams. The prioritized scheme not only allows a user to specify priority on multiple selected streams but also enables traffic engineering routing paths for optimal usage of network bandwidth. 4.) Application supports for service differentiation MQRP allows a receiver to accept a partial allocation of the full stream bandwidth. The partial bandwidth allocation strategy not only reflects the heterogeneity of host capability but also provides the flexibility for network provider to maximize the overall utilization.

\section{PRIORITY-DIFFERENTIATED DELAY-BOUNDED MULTICAST}

Formulation of the Problem. Given a network with a multicast group $G$, the source node $s$ announces a set of streams with specification $T_{\text {spec }}$ to all network nodes. A potential receiver $d$ in $G$ requests multiple streams by specifying a priority vector $P(d)$ and an associated delay bound $\Delta(d)$. The problem is to find a multicast tree $T$ connecting all the requesting receivers and to allocate bandwidth $R^{j}(u, v)$ for each stream $j$ on each link $(u, v) \in T$ so as to satisfy $\Delta(d)$ for the admitted streams. On establishing such tree, it is the objectives to maximize the gain over these requests and minimize the tree cost, in order to leverage the utilization and efficiency of the network resources. Specifically, the utilization of the network resource (bandwidth) 
$\Upsilon$ is defined as $\Upsilon=\frac{T S}{\text { Cost }}$, where $T S=\sum_{(u, v) \in T, \forall j} 1_{\left[R^{j}(u, v) \neq 0\right]}$, is the total number of streams that has been admitted to the multicast. (The indication function $1_{[x]}$ returns one if $x=$ TRUE). The tree cost is defined as the total amount of bandwidth that has been allocated to the multicast tree $T$, i.e., Cost $=\sum_{(u, v) \in T, \forall j} R^{j}(u, v)$, for all $R^{j}(u, v) \neq 0$. Likewise, the efficiency is defined as $\Theta=\frac{G a i n}{M G}$, where Gain, the sum of the priorities of all the admitted streams, i.e., $\sum_{d \in G, \forall j} P^{j}(d), R^{j}(u, d) \neq 0$, and $M G=\sum_{d \in G, \forall j} P^{j}(d)$, is the maximal gain that can be possibly obtained from all the requests.

An Overview of the Protocol. MQRP assumes that the link latency information is propagated over the network via a link state routing protocol (e.g., OSPF); thereby, each node has the information of global network topology and hence can derive the minimum path latency (MPL) to all othe $r$ nodes. The signaling procedure of MQRP is depicted in Figure 1. The protocol proceeds in three phases, with respect to the signaling phases of RSVP. First, during the stream announcement phase, the source announces the stream specification $T_{\text {spec }}$ by sending a RSVP PATH message via the minimum latency paths downstream to each destination.

Next, during the tree establishment and resource reservation phase, each node willing to participate the multicast session specifies the $R_{s p e c}$ in a RSVP request message RESV, indicating the priorities and delay bounds requested. These RESV messages are sent upstream towards the source node. On receiving the messages, intermediate nodes merges the requests and connect to an upstream node using the link latency information offered by link state routing table and the information contained in $R_{\text {spec }}$ and $T_{\text {spec }}$. The phase ends when the source receives all the RESV messages, when the tree is established with bandwidth $R$ reserved on each link for each stream.

Finally, during the admission control and resource allocation phase, the source exerts admission control to the requesting streams and starts to allocate bandwidth for each stream by sending RSVP PATH Refresh messages toward all receivers via the tree tha $t$ has been established in previous phase. On receiving the PATH Refresh message, each node extracts the information in the Refresh message and calculate the amount of bandwidth $R$ to reserve for each stream to be admitted to the multicast.

\section{MULTICAST QOS-BASED ROUTING AND RESERVATION}

The algorithm for MQRP consists of two parts: one for multicast tree establishment and resource reservation and the other for admission control and bandwidth allocation. The former reserves bandwidth when establishing the 


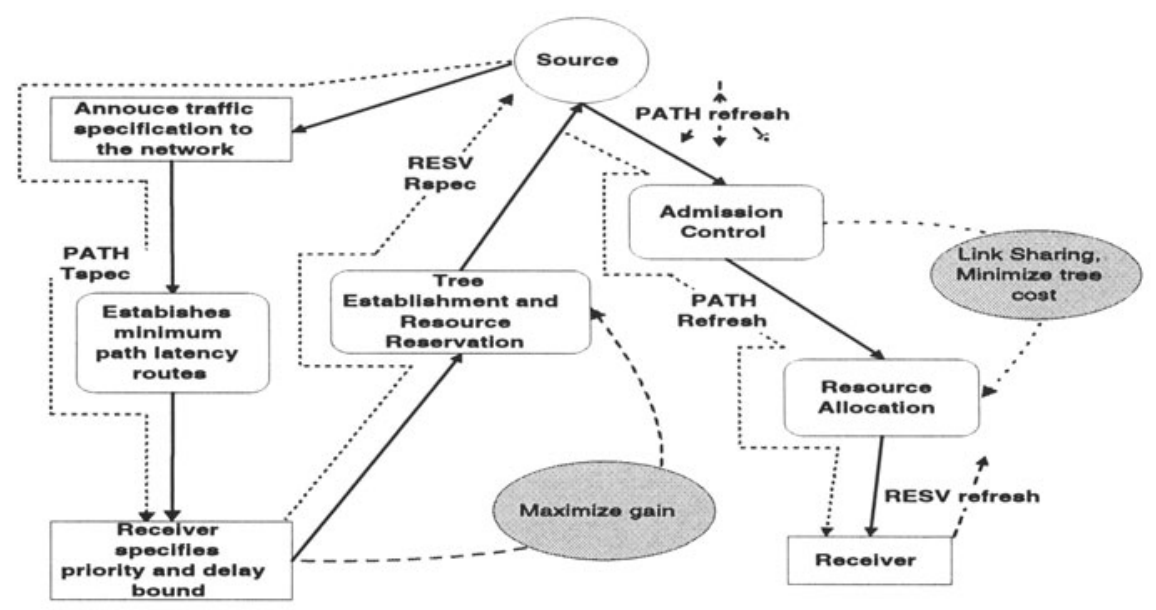

Figure 1 Signals in the integrated reservation and multicast protocol

tree, whereas the latter performs admission control and allocates the bandwidth for each stream.

\subsection{MULTICAST FLOW AND DELAY MODEL}

The operation of MQRP is based on the admission control policy of multicast flows and on a delay model. The algorithm for multicast tree establishment starts with merging requests from destinations with distinct flow requirements.

a peak rate of 35 via a by successfully. In the example,

eive the stream in peak rate so that it acking of bandwidth, but they still can participate the multicasting by

Delay model from node $s$ to nodes $d_{1}$ and

The delay model used in MQRP is approximated by the parameters in the traffic specification and the delay error terms incurred in each router, as specified in the perfect fluid model [7][8]. According to the fluid model [13], the end-toend queuing delay can be computed via the traffic parameters in $T_{s p e c}$, which include the peak rate of the flow $(p)$, the token bucket depth $(b)$ and rate $(r)$, the minimum policed unit $(m)$, and the maximum datagram size $(M)$ [4]. Assuming that a fixed amount of latency is associated with each transmission link, then the end-to-end delay from source $s$ to a destination $d, D(s, d)$, is equal to the end-to-end queuing delay $Q(s, d)$ plus the fixed amount of path latency $P L(s, d)$ from $s$ to $d$, i.e.,

$$
D(s, d)=Q(s, d)+P L(s, d)
$$


While the path latency is fixed, the queuing delay will vary as the bandwidth assigned to the path changes. This fluid model explicitly shows that a stream conforming to a token bucket will increase its queuing delay as the allocated path bandwidth decreases [4]. If a bandwidth $R(u, d)$ from node $u$ to a destination $d$ is reserved and is greater than or equal to the peak rate $p$, i.e., $R(u, d) \geq p$, then the queuing delay $Q(u, d)$ can be estimated by

$$
Q(u, d)=\frac{M+C_{t o t}}{R(u, d)}+D_{t o t}
$$

Otherwise, for $r \leq R(u, d)<p$, it is computed as

$$
Q(u, d)=\frac{(b-M)(p-R)}{R(u, d)(p-r)}+\frac{M+C_{t o t}}{R}+D_{t o t},
$$

where $D_{t o t}$ and $C_{t o t}$ account for the sum of error terms in each routers along the path.

be admitted to the multicast

\subsection{TREE ESTABLISHMENT AND BANDWIDTH RESERVATION}

The MQRP protocol is represented as a distributed algorithm operating at each node. As MPL is derived from PATH messages, each node then initialize two parameters: aggregated priority $\bar{P}$ and admissible path delay $\bar{\Delta}$. The aggregate priority $\bar{P}(i)$ at node $i$ is the sum of the priority values of all streams in the current tree. The admissible path delay $\bar{\Delta}(i)$ represents the latest time a packet should arrive at this node $i$. If a packet arrives at this node with a delay larger than $\bar{\Delta}(i)$, then it is impossible for the packet to be delivered to the destination within specified delay bound.

PROCEDURE Multicast QoS Routing and Reservation Protocol

1 If receive the PATH message Compute $M P L(d), \forall d \in V$

2 For each destination $d \in G$

$$
\bar{P}(d)=P(d), \quad \bar{\Delta}(d)=\Delta(d), R(d)=p(d)
$$

3 If receives the RESV message

$$
\begin{aligned}
& \bar{\Delta}(v)=\operatorname{Min}_{d}\left\{\bar{\Delta}_{d}(v)\right\}, \bar{P}(v) \leftarrow \bar{P}(v)+\bar{P}(w) \\
& R(v)=\operatorname{Max}_{w}\{R(v, w)\}, \forall w \in \operatorname{Child}(v)
\end{aligned}
$$

4 For each $u, M P L(u)<M P L(v), M P L(u)+L(u, v)<\bar{\Delta}(v)$

Calculate $\rho(u, v)=\operatorname{Min}\left(\frac{C(u, v)}{S_{\text {sum }}(v)}, 1\right)$

$$
S_{s u m}(v)=\sum_{j} R^{j}(v, w),\left\{j \mid \bar{P}^{j}(v) \neq 0\right\}
$$

Estimate $R(u, v)=R(v) \rho(u, v)$

For each destination $d$ 


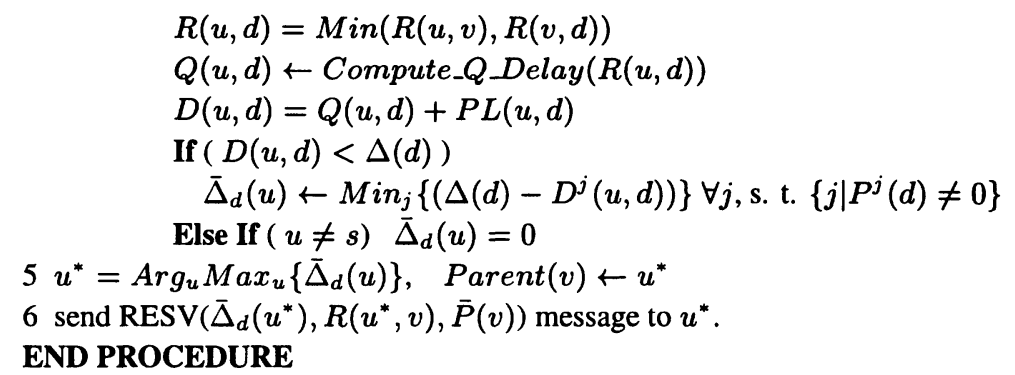

The tree establishment phase is initiated from the receivers by sending RESV messages to the source. A node $v$ extracts $\bar{\Delta}_{d}(v), R(v, w)$ and $\bar{P}(w)$ carried in the RESV message from a downstream node $w$, where $\bar{\Delta}_{d}(v)$ is the admissible delay at node $v$ for destination $d$ and $R(v, w)$ is the bandwidth reserved on link $(v, w)$. For a number of destinations requesting the stream via node $v$, the smallest admissible delay must be selected for $v$. That is, $\bar{\Delta}(v)=$ $\operatorname{Min}_{d}\left\{\bar{\Delta}_{d}(v)\right\}$. Also, node $v$ must select the largest bandwidth among these requests merging to $v$ as the bandwidth $R(v)$ to serve on the upstream link. That it, $R(v)=\operatorname{Max}_{w}\{R(v, w)\}$, for all the children $w$ 's of node $v$.

To reserve the bandwidth, node $v$ needs to find an optimal nodes $u^{*}$ to connect, among the neighboring nodes. The neighboring node $u$ must satisfy two conditions: $M P L(u)<M P L(v)$ and $M P L(u)+L(u, v)<\bar{\Delta}(v)$. The minimum path latency from source to $v$ via node $u$ must satisfy, $M P L(u)+$ $L(u, v) \leq \bar{\Delta}(v)$; otherwise, it is impossible to deliver the streams to node $v$ in time. Besides, if $v$ request the bandwidth $R(v)$ on link $(u, v)$ that is greater than the capacity $C(u, v)$, then it is impossible to deliver all the streams at $R(v)$. In such case, in order to accommodate as many streams as possible on link $(u, v)$, the bandwidth requirement must be reduced by a fraction $\rho(u, v)$ such that the bandwidth requirement for link $(u, v)$ becomes $R(u, v)=R(v) \rho(u, v)$.

According to the fluid delay model, the queueing delay (and thus the endto-end delay) is determined by the path bandwidth (the bandwidth of smallest link among a path), $R(u, d)$. Hence, queuing delay $Q(u, d)$ can be estimated according to Equation 1 or 2 . As a result, the end-to-end delay from node $u$ to destination $d$ can be obtained through $D(u, d)=Q(u, d)+P L(u, d)$.

Now, the admissible delay $\bar{\Delta}_{d}(u)$ at node $d$, where $D(u, d)<\Delta(d)$, is updated to $\operatorname{Min}_{j}\left(\Delta(d)-D^{j}(u, d)\right)$, for all $j$ 's in $d$ such that $P^{j}(d) \neq 0$. Having all the admissible delays computed at all neighboring node, node $v$ selects the optimal neighboring $u^{*}$ by

$$
u^{*}=\operatorname{Arg}_{u} \operatorname{Max}\left\{\bar{\Delta}_{d}(u)\right\},
$$

where " $\mathrm{Arg}_{u} \mathrm{Max}$ " returns the corresponding index $u$ that produces the largest $\bar{\Delta}_{d}(u)$ value. Finally, node $v$ will sends upstream node $u^{*}$ a RESV message 
that contains $\bar{\Delta}_{d}(u), R\left(u^{*}, v\right)$, and $\bar{P}(v)$ to node $u^{*}$. When receiving the RESV message from node $v$, node $u^{*}$ will reserve the amount of bandwidth $R\left(u^{*}, v\right)$ on link $\left(u^{*}, v\right)$ and use $\bar{\Delta}_{d}(u)$ and $P_{d}(v)$ to select a neighboring node to connect, repeating the above procedure.

\subsection{ADMISSION CONTROL AND RESOURCE ALLOCATION}

The admission control and resource allocation algorithm allocates bandwidth to each tree link. This algorithm starts from the source node when it received all the RESV messages from the destinations and then iterates downstream to the destination nodes. When a node $v$ receives a PATH Refresh from its upstream node, it extracts the $P L(s, d)$ and estimate the queueing delay according to $Q(s, d)=\Delta(d)-P L(s, d)$. Then, it estimates the amount of bandwidth required for this queueing delay using Equation 1 or 2 . This bandwidth, $\tilde{R}$, will be further redistributed among the streams in order to maximize the total gain. The function Band_Share () returns the optimal distribution of the bandwidth $R(v, d)$ for maximal gain. Suppose that $w$ is a child of $v$ which lead the streams from $v$ to all destinations. For each stream, node $v$ reserves the amount of bandwidth that is maximum of the bandwidths required by all destination along the link $(v, w)$ so that all the destinations can receive the stream. Specifically, $v$ reserves $R(v, w) \leftarrow \operatorname{Max}_{d}\{R(v, d)\}$. Finally, node $v$ allocates a bandwidth on the link $(v, w)$ for each stream by first selecting the stream with largest priority and repeating until the link capacity is exhausted.

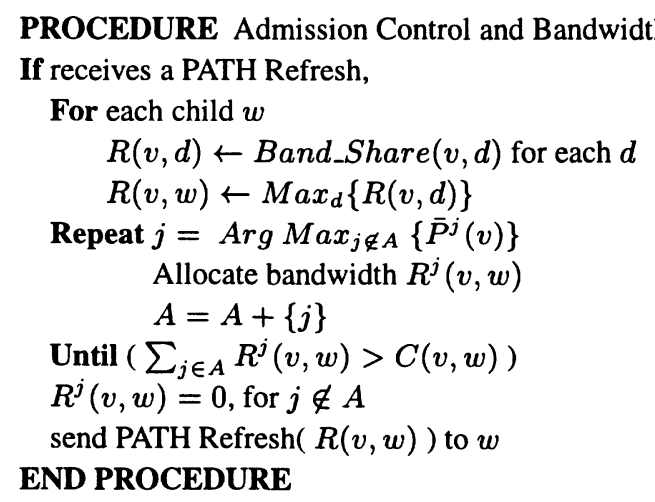

\section{SIMULATION}

The performance of the protocol will be evaluated in terms of two measures: utilization and efficiency, as defined earlier. During simulations, focused are the performance impacts due to change of the metrics including the network 
size, percentage of the multicast membership, total number of streams, delay offset, and bandwidth sufficiency. The delay offset is defined as the specified delay bound minus the calculated path latency, $\triangle-M P L$, which accounts for the time interval that can be used to trade for more streams to join. The bandwidth sufficiency, a quantity that indicates how sufficient the network capacity is, compared to the stream bandwidth specification, is defined as $\frac{\frac{1}{|E|} \sum_{(u, v) \in E} C(u, v)}{\frac{1}{q} \sum_{j=1, \ldots, q} S_{j}}$, where $|E|$ is the number of links in the network.

Several protocols are compared in the simulations. In addition to the MQRP protocol, we also derive two its variants that allocate bandwidth along the path without trimming the tree on phase three: the MQRP-Total and the MQRPPartial. The partial reservation scheme allows reserving a portion of the peak rate with reduced media quality. The P-RSVP is a modified version of RSVP for accommodating the priority on receiver request. The NHP3 is the third procedure of the four proposed procedures in [2], but the authors do not specify the tree structure in the work. We modify the third procedure in their work into two variants: maximum capacity tree (NHP3-1) and minimum cap acity tree (NHP3-2).

sign the link bandwidth in

\subsection{RESOURCE UTILIZATION AND SCALABILITY}

To study the robustness and scalability, protocols are evaluated on a class of random network [14] that have dynamically changed topology and are relatively large scale. In the experiment, we gradually scale the network to 200 nodes while keeping the average node degree to 3 , and measure the utilization of the network with a given fixed bandwidth sufficiency. It means that the ratio of network capacity to stream bandwidth remains constant regardless that the network grows. Of the network nodes, 40 percent are designed as the receivers. Figure 2 shows the result that MQRP protocol is more scalable than the other algorithms. The scalability of MQRP protocol is about four times that of the P-RSVP. In Figure 3, we find that the utilization increases as the number of streams increases. The utilization produced by the MQRP scheme increases in a rate about four times that of the NHP3-1 scheme and about two times that of the P-RSVP scheme. This demonstrates the capability of MQRP protocol on accommodating more number of streams. The bandwidth reservation scheme in MQRP can admit more steams to the multicast when all the protocols running in a network with scarce resource.

est path delay observed at o that the overall utilization and efficiency are leveraged. 


\subsection{GAIN AND BANDWIDTH EFFICIENCY}

The efficiency is determined by the total gain that can be anticipated. As the network scale from 20 nodes to 200 nodes, the MQRP and P-RSVP protocols are very competitive, as shown in Figure 4 . This shows that the P-RSVP is also an efficient protocol because the receiver always adopts the shortest path delay to the source without considering the priority in establishing the multicast tree. Although it accommodates more streams to enter; however, it will not guarantee the best streams (streams with high priority) are admitted. Hence, as shown in Figure 2 the utilization degrades. The protocols with the worst efficiency are NHP3-2 and MQRP-Partial. The NHP3-2, although establishing the tree by considering the priority, finds a minimum capacity tree, which limits the number of streams to be admitted into the multicast tree, resulting in poor utilization.

As a result, it may not be able to tial has the worst efficiency. But, as shown in Figure 3, it still In Figure 5, we compare the efficiency by varying the bandwidth sufficiency from 0.2 to 1.2. MQRP protocol has the best efficiency among them, whereas the NHP3-2 has the worst when the bandwidth sufficiency is below 2. But the MQRP-Partial become s the worst when the bandwidth sufficiency is above 2 . This shows that MQRP is suitable for the network with relatively heavy-load or the network with scarce capacity relatively to the total requested bandwidth of the receivers.

\section{CONCLUSION}

In this article, we have shown that the proposed MQRP is able to optimize the bandwidth utilization and efficiency for network resources whilst satisfying user preferences and constraints. From the simulation results, it is also shown that MQRP is able to scale best with the number of network nodes as well as offered streams. To sum up, MQRP as a QoS-based routing and reservation multicast protocol is able to accommodate heterogeneous application requirements to support prioritized delay-bounded multicast service, enabling the network provider to optimize the resource utilizat ion and to gain the revenue efficiently from the provisioning.

\section{References}

[1] G.N. Rouskas, and I.Baldine, "Multicast Routing with End-To-End Delay and Delay Variation Constrains", IEEE Journal on selected areas in communications, Vol. 15 , No. 3, April 1997, pp.346-356.

[2] N. Shacham and James S. Meditch, "An Algorithm For Optimal Multicast of Multimedia Stream,", in Proceedings of IEEE INFOCOM'94, Vol. 2, pp.586-64. 
[3] V.P. Kompella, J.C. Pasqale, and G.C. Polyzos, "Multicast Routing for Multimedia Communication", IEEE/ACM Transactions on Networking, 1993.

[4] L. Zhang, S. Deering, D. Estrin, S. Shenker and D. Zappala, "RSVP:A New Resource ReSer Vation Protocol", IEEE Network, Sept. 1993.

[5] S. Schenker, C. Partridge, and R. Guerin, "Specification of Guaranteed Quality of Service", IEEE Communications Magazine, May 1997.

[6]D.J. Mitzel, D. Estrin, S Shenker and L. Zhang. , "An Architectural Comparison of ST-II and RSVP", IEEE 1994.

[7]A. Parekh and R. Gallagher, "A Generalized processor Sharing Approach to Flow Control - The Single Node Case," IEEE/ACM Trans. Networking, vol. 1, no. 3, 1993, pp. 366-57.

[8]A. Parekh and R. Gallagher, "A Generalized processor Sharing Approach to Flow Control - The Multiple Node Case," IEEE/ACM Trans. Networking, vol. 2, no. 2, 1996, pp. 137-50.

[9]E. Crawley, R. Nair, B. Rajagopalan, and H. Sandick, " A Framework for QoS-based Routing in the Internet," RFC 2386, August 1998.

[10]Zhang, Z., Sanchez, C., Salkewicz, B., and E. Crawley, "QoS Extensions to OSPF", Work in Progress.

[11]Zappala, D., Estrin, D., and S. Shenker, "Alternate Path Routing and Pinning for Interdomain Multicast Routing", USC Computer Science Technical Report \# 97-655, USC, 1997.

[12]Y. Goto, M. Ohta and K. Araki, "Path QoS Collection for Stable Hop-byHop QoS Routing", Proc. INET '97, June, 1997.

[13] S. Shenker, C. Partridge, and R. Guerin, "Specification of Guaranteed Quality of Service," RFC 2212, September 1997.

[14] B. M. Waxman, "Routing of Multiple Connections," IEEE Journal on selected areas in communications, Vol. 6, No. 9, December 1988, pp.16171622. 


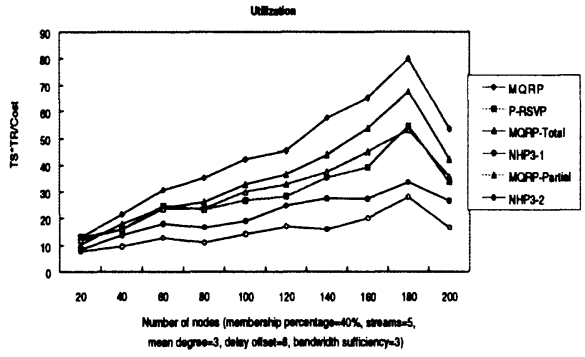

Figure 2 Utilization versus the network size

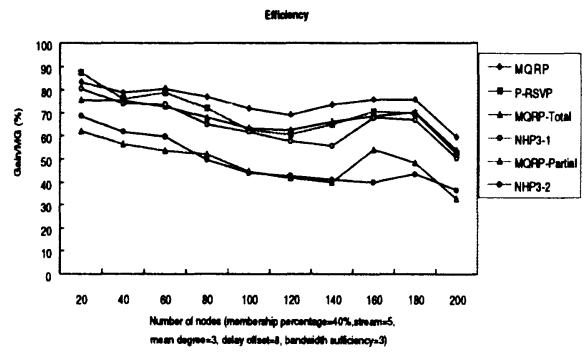

Figure 4 Efficiency versus the network size

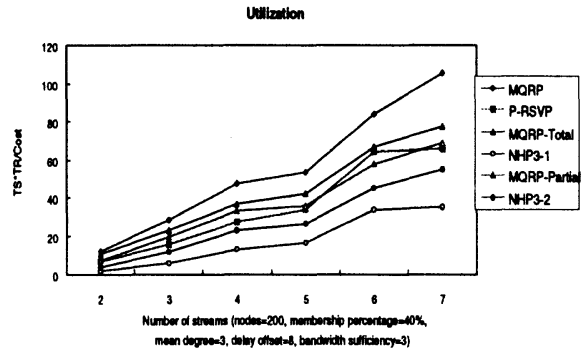

Figure 3 Utilization versus the number of source streams

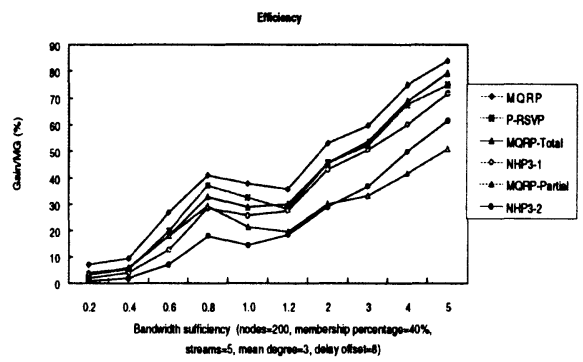

Figure 5 Efficiency versus bandwidth sufficiency 